\title{
Elfe XX-XXI
}

ELFe XX-XXI

Études de la littérature française des XXe et XXIe siècles

$7 \mid 2019$

Littérature et cuisine

\section{Une histoire littéraire de la cuisine. Rassasier le lecteur : éloge d'une littérature jubilatoire}

Entretien avec Arno Bertina

Arno Bertina, Johan Faerber et Nathalie Vincent-Munnia

\section{OpenEdition}

Journals

Édition électronique

URL : http://journals.openedition.org/elfe/493

DOI : $10.4000 /$ elfe.493

ISSN : 2262-3450

Éditeur

Société d'étude de la littérature de langue française du XXe et du XXle siècles

Référence électronique

Arno Bertina, Johan Faerber et Nathalie Vincent-Munnia, « Une histoire littéraire de la cuisine. Rassasier le lecteur : éloge d'une littérature jubilatoire », Elfe XX-XXI [En ligne], 7 | 2019, mis en ligne le 01 avril 2019, consulté le 21 septembre 2020. URL : http://journals.openedition.org/elfe/493 ; DOI https://doi.org/10.4000/elfe.493

Ce document a été généré automatiquement le 21 septembre 2020.

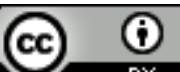

La revue Elfe $X X-X X I$ est mise à disposition selon les termes de la Licence Creative Commons Attribution 4.0 International. 


\title{
Une histoire littéraire de la cuisine. Rassasier le lecteur : éloge d'une littérature jubilatoire
}

Entretien avec Arno Bertina

\author{
Arno Bertina, Johan Faerber et Nathalie Vincent-Munnia
}

NATHALIE VINCENT-MUNNIA : Après Le Dehors ou la migration des truites puis Appoggio, La Déconfite gigantale du sérieux se présente, en 2004, comme une pseudo-autobiographie de Pietro Di Vaglio. Ce jeune romantique de I'Italie des années 1810-1820 finit par découvrir une auberge rabelaisienne, peuplée d'une aubergiste gargantuesque et de personnages à la langue débraillée, populaire, mais ô combien plus riche et vivante pour lui que celle du petit cénacle dans lequel, jusque-là, il se prenait au sérieux - mais qui « tourne à la farce (sans viande) »'. Par-delà ce parcours initiatique du jeune Pietro Di Vaglio, les notes de bas de pages prennent progressivement de plus en plus de place au fil du récit et dévoilent une véritable esthétique. L'une d'elles évoque justement Rabelais ${ }^{2}$. Serait-il possible de préciser cette vision rabelaisienne de la littérature défendue dans La Déconfite du sérieux, que tu vises aussi dans ta propre écriture?

Arno Bertina : Ce petit livre est une sorte de manifeste personnel déguisé. Je ne voulais pas écrire un manifeste, un art poétique, mais cela m'amusait de le faire passer par le biais d'une fiction. C'est mon troisième livre, et depuis trois ou quatre ans, au cours des invitations, j'écoutais d'autres auteurs parler de leur rapport à l'écriture, à la littérature, qui continuaient de véhiculer des poncifs sur la création, tous marqués par une conception très romantique de l'artiste, de l'écrivain, de l'écriture : la douleur d'exister - on écrirait pour lutter contre la douleur d'exister dans la lignée de Flaubert - alors que, quand vous lisez ensuite les textes de ces auteurs, vous ne voyez vraiment pas en quoi la virgule a été vraiment travaillée, elle est juste académique... Tout ce qui restait du discours romantique, d'une pensée romantique de l'auteur chez les écrivains d'aujourd'hui, je voulais à tout prix me le payer, je voulais me moquer de ça. Mais je ne voulais pas me moquer de ça dans le cadre d'un pamphlet: ça ne m'intéressait pas de les nommer, ces auteurs-là puisqu'ils ne m'intéressent pas, je ne vais pas les nommer - mais je voulais essayer de débarrasser l'écriture de cette espèce de chape de sérieux qui l'entoure. Et donc, 
quand on cherche à faire ça, évidemment, un des tout premiers repères qu'on trouve, c'est d'en passer par Rabelais. C'est par ce biais-là que ce livre est devenu quelque chose de plus intelligent qu'un pamphlet, c'est-à-dire une sorte de livre d'amour, de déclaration d'amour pour Rabelais. Je m'inscris là évidemment dans une tradition : des auteurs et des lecteurs qui ont dit leur amour pour Rabelais, il y en a des milliers. Mais j'avais l'impression que le texte de Rabelais avait quelque chose de très acide par rapport à notre époque. Je pourrais le résumer à une seule opposition : il y a une phrase dans Rabelais, qui est une phrase magnifique, merveilleuse, où il dit qu'il préfère que l'on dise de ses livres qu'ils sentent le vin et non pas l'huile. Et quand il dit « l'huile », ça désigne l'huile de la lampe à huile, c'est-à-dire de l'auteur besogneux qui va écrire toute la nuit, qui va travailler... Il y a donc déjà cette figure du lettré besogneux. Cette notion de travail du texte va être sur-mise en scène par Flaubert, notamment dans sa correspondance: une ligne dont je voulais me détacher, pour m'inscrire dans les pas d'une autre énergie. Et tout ça, c'est une ambition : je ne peux pas du tout prétendre que mes livres réussissent cette ambition, mais c'était dans cette direction que je regardais.

NATHALIE VINCENT-MUNNIA : Johan Faerber parle d'écriture baroque, en particulier à propos d'Appoggio - et du personnage féminin d'Appoggio : cette cantatrice, complexe et multiple, qui a plusieurs noms, qui a plusieurs identités, notamment dans son rapport avec la musique. Es-tu d'accord avec ce terme de baroque concernant Appoggio ? Ou en tout cas si ce n'est le terme, sur ce qu'il recouvre: une écriture de la jubilation - un peu le même propos que La Déconfite du sérieux - où il est surtout question de corps... Je vais lire un passage d'Appoggio, où il est question du rapport à la nourriture de ce personnage féminin, et qui témoigne aussi de l'inscription de son corps dans l'espace et dans sa relation avec les autres.

[À propos de la cantatrice] Et c'est même la première chose qu'ils remarquent, ce rire qui vous porte et les déplace, leur enlève de la place à eux pour respirer. Et la sauvagerie avec laquelle elle se jette sur le buffet, puis sur les plats, ils la remarquent aussi et ils s'accrochent à ça, sur cela ils ont prise parce qu'elle s'oublie complètement, visiblement [...]. «Quel appétit!» dit un con. «La nourrissez-vous seulement?» renchérit un autre, qui pour être spirituel s'essaya au parler désuet. Mais à ce moment, on apporta du poulet froid. Elle arracha une cuisse au bestiau, je me régalais, les autres s'arrêtèrent un peu gênés car elle mangeait bruyamment maintenant. Et quand elle eut fini de sucer les os qu'elle enfonçait jusqu'au fond de la gorge comme on le fait avec un bâtonnet d'esquimau, elle regarda tout le monde avec des yeux rieurs. Elle exultait, tout entière au plaisir qu'elle venait de prendre en dévorant cette aile et cette cuisse, ne songeant pas même à utiliser sa serviette pour gommer les taches luisantes qu'elle s'était faites sur les joues et jusque sous les yeux. Elle jubilait. Puis, ne respectant pas l'ordre du repas, elle s'en prend aux choux à la crème, dont on retrouva quelques larmes sur son corsage, elle fait son affaire au fondant à la poire. Des salades traînaient par là, des poivrons marinés qui font des couleurs dans les pâtes, ou des lasagnes dont elle racla le plat pour ne pas laisser aux piafs les bords gratinés dit-elle en se jetant dessus.

Mable était accessible à cette joie-là. Pour moi c'était de la vie, et non de la boulimie, c'était de la vie et non pas une névrose. ${ }^{3}$

Peux-tu nous parler de ce récit, Appoggio, de ce personnage, de son corps?

Arno Bertina : Appoggio est un terme qui existe en musique, qui n'est pas passé dans le langage courant en français, mais qui relève d'un langage très courant en Italie, et qui désigne l'appui. En musique, on invite les chanteurs à chercher un appui, qui est à la fois un appui physique pour la colonne d'air, et aussi un appui mental depuis lequel les chanteurs vont arriver à projeter la voix. Et donc mon personnage dans le roman est à la recherche de cet appui-là. Elle n'est pas sereine dans son corps. Comme dans 
cette scène, son corps la dépasse en permanence et donc ça lui pose problème, ça ne fait pas d'elle une grande chanteuse. Elle est trop instable pour prétendre dominer son art, donc elle est à la fois fascinée par ce qui se joue sur une scène d'opéra, et par le statut, par la place qui est faite au corps dans le chant, c'est-à-dire quelque chose de complètement fou, mais elle est malmenée par cette puissance-là. À la toute fin, elle quitte tout le monde et elle part en Italie parce qu'elle a appris qu'à Bologne, dans les années 1890 je crois, il y avait une très grande cantatrice - dont j'ai oublié le nom-native de Bologne, qui avait légué son larynx à la mairie de Bologne. On estimait que si elle avait été une grande chanteuse, c'était justement du fait de ce larynx, et que c'était cela qui valait de l'or et qu'il fallait à tout prix préserver du tombeau. Elle erre donc dans Bologne, à la recherche de ce bocal qui doit bien être quelque part, parce qu'elle n'imagine pas un fonctionnaire municipal décider un jour de balancer le larynx en question - et pourtant ça ne doit pas être ragoûtant... Et elle est sûre, si elle le trouve, d'un truc un peu magique, d'une pensée magique. Si elle le trouve, elle va trouver une sorte d'explication, comme si elle pouvait accéder enfin à quelque chose qui la pacifierait. Donc elle est là... Quand elle chante, il se passe quelque chose en elle, qui est dans le ventre, la poitrine, le cou, etc. : mais elle est dans cette pensée magique, elle ne sait pas où ça se passe, et ce qui se passe exactement. Résultat, tout ce qui peut venir combler ça, que ce soit par la nourriture, que ce soit par une sorte de débauche sexuelle, ou dans le fait d'être très agressive avec les autres, c'est-à-dire d'aller les chercher physiquement, il y a toute sa détresse qui s'exprime à ce moment-là. Quand Johan parle de la dimension baroque - ça prend appui sur sa propre thèse qui examinait les rapports entre le Nouveau Roman et l'esthétique baroque -, c'est en désignant un autre aspect du livre: tous ces emboîtements d'identités, tous ces jeux de faux-semblants qui sont à l'intérieur du livre et qui sont en contraste avec cette épaisseur physique qui elle, au contraire, l'obsède. Il y a d'un côté cette dimension survivante du personnage, ou en tout cas que j'ai espérée survivante - au sens de super-vivante - et de l'autre côté, elle est cernée par des abîmes. Tout d'un coup, elle prend un personnage parce qu'elle est dans telle ou telle distribution d'opéra, donc elle est un personnage, mais ensuite elle doit le quitter, et ce sont des choses qui ne se font pas facilement pour elle... Donc cette dimension baroque, c'est justement une dimension de jeu à laquelle elle n'arrive pas à accéder. Elle est prise dans une sorte de vertige alors qu'il y a moyen - et c'est ce que dit en permanence l'esthétique baroque - il y a moyen de gagner là-dedans une liberté, en n'ayant pas peur de la mort, en intégrant la mort, en comprenant que tout n'est fait que de métamorphoses et de passages. Elle, au contraire, elle est trop instable pour arriver à jouir de ça.

NATHALIE VINCENT-MUNNIA : Johan Faerber, dans son analyse d'Appoggio, dit que ce personnage glisse de l'auxiliaire être - de l'auxiliaire est - à la conjonction et, sans arriver à construire une identité stable et définitive, tout comme l'intrigue ne se construit évidemment pas linéairement. Ce personnage, Mable, Ariane - encore une Ariane - est dans l'excès, l'excès du corps. C'est quelque chose qui revient dans d'autres récits, dans Anima motrix, dans Je suis une aventure, avec - à l'horizon de nos préoccupations culinaires - tous ces dérèglements du corps, qui peuvent être dus à la nourriture, au sexe, à l'alcool...

Arno Bertina : À chaque fois, il y a la même envie de faire vivre quelque chose que je ne trouve pas si souvent que ça, dans la littérature française s'entend. Pascal Quignard fait une remarque très curieuse dans un des petits traités : il parle de ces génies littéraires qui n'ont pas eu d'enfants. Par exemple, de manière significative, 
tout le monde, quasiment à toutes les époques, fait de Rabelais un phare de la littérature, mais en fait il y a très peu d'auteurs qui ont voulu vraiment s'inscrire dans ses pas... et on pourrait trouver d'autres exemples : il est difficile de trouver des continuateurs à Stendhal, malgré les Hussards... alors que les continuateurs de Flaubert sont très nombreux. Cet aspect de la littérature française, souvent bizarrement un peu anémiée, me chiffonne.... Il y a un autre livre dont je suis très fan, Le Moyen de parvenir, qui n'est pas un texte très connu, mais qui a toujours été publié, à toutes les époques, a toujours été disponible - aujourd'hui encore, il l'est en poche et en grand format -: c'est un banquet, un banquet incroyable, il y a quatre cents convives qui sont réunis. Il y a aussi bien Jules César, une boulangère, François $\mathrm{I}^{\mathrm{er}}$, Ronsard...Tous prennent la parole en même temps : Ronsard, c'est évidemment pour sortir des blagues d'une obscénité absolument invraisemblable; il y a Périclès qui bégaye en permanence alors qu'on nous le vend comme le grand orateur politique, etc. Et puis il y a des gens du peuple, tout est très mélangé... Ils prennent tous la parole une ou deux fois, et cette parole ne tourne qu'autour de repas, de jeux de mots, de sexe aussi, et il y a une vitalité folle là-dedans. Pour moi, le fait d'écrire, c'est une expérience de la joie, et donc naturellement ça en devient aussi le sujet. Je trouvais assez fantastique ce que disait Johan Faerber quand il disait que BrillatSavarin avait dû en passer par l'invention de quantité de termes... Avec le corps qu'on essaie de faire résonner dans des milliers de directions, il y a très vite ce côté feu d'artifice de la langue. Et j'ai le sentiment que pour bousculer les représentations, pour ne pas être toujours dans la réécriture du même livre, il faut en passer par cette dimension très physique. Essayer de faire qu'on sorte de cette espèce d'équilibre très français, très classique, qu'il y a dans quatre-vingt-dix pour cent de la production littéraire. Donc, oui, j'essaie dans presque tous les livres de faire vivre cette part-là, qui est du langage, et qui est aussi dans le regard. Curieusement, ce n'est pas très facile. Par exemple, dans Je suis une aventure, le narrateur est un journaliste, qui n'a qu'une chose en tête, en permanence : ce sont les femmes. Et c'était assez incroyable de voir les retours de nombreux lecteurs venant me dire : "Il est obsédé ». Non, il n'est pas obsédé, il est vivant. Obsédé, ça veut dire qu'il y a un problème. S'il y a un problème, montrez-le-moi : si vous le trouvez phallocrate, misogyne, montrez-le-moi. Or, je l'ai dit à chaque fois au début, parce que je sais très bien que ce n'est pas le cas : je ne crois pas qu'il y ait une seule pensée ou façon de voir qui serait prédatrice ou consumériste. Non, c'est être vivant, c'est être dans des rapports de séduction - avec les hommes, avec les femmes, peu importe, ce n'est pas la question -, être dans des rapports de séduction, avoir envie du corps de l'autre, et trouver dans le corps de l'autre, dans la présence de l'autre, des ressources pour la joie absolument incroyables. La place du corps dans la littérature française n'est pas toujours si simple que ça... Encore une fois, je vais vite en disant ça, et je ne prétends absolument pas être le seul à essayer de travailler cette question-là, mais ça me surprend toujours à quel point elle reste prude malgré de très grands ancêtres.

NATHALIE Vincent-MUNNiA : Donc la cuisine doit être grasse, la littérature doit être grasse, et l'amour, le sexe évidemment, doivent être gras aussi...

JOHAN FAERBER : Ce qui m'a frappé quand j'ai lu Appoggio (et là, ce n'est plus tellement la cuisine qui se mange, c'est plutôt la cuisine littéraire), c'est le caractère de rupture totale avec le premier roman: Le Dehors ou la migration des truites. Alors que le premier représentait, pour dire les choses vite, le massacre du métro Charonne, le deuxième est beaucoup plus dans la joie. II a beaucoup plus d'appétit, et un double appétit: autant le premier était l'histoire d'une voix qui courait d'homme en homme et de femme en femme, 
autant celui-là est vraiment une histoire du corps, il s'incarne. II a un véritable appétit, de manger, de vivre, mais aussi un appétit narratif, avec vraiment beaucoup d'inventivité, et pas cette espèce de formalisme un peu bon teint qu'on voit un peu, où, pour écrire, il faudrait trouver une forme, etc. Et ce qui est formidable, c'est qu'on donne quelque chose qui est de l'ordre de la nourriture. Appoggio nous livre à l'informe: il y a une science de l'informe, on essaie de tracer des lignes directrices dans l'informe, et c'est ce qui met, comme un repas, en joie...

Arno Bertina : Dans Anima motrix, on est dans un livre de métamorphose, on suit un personnage qui est en fuite et qui se transforme, - je tisse cela jusqu'au bout -, qui se transforme en cerf, quasiment entre le début et la fin du roman. Donc là c'est le corps qui échappe à l'identité, c'est-à-dire l'identité telle qu'on la connaît, entre humains. Essayer de montrer que tout d'un coup, dans le corps, peuvent se jouer des choses cent fois plus vastes que ce qu'on imagine. Il y a aussi une dimension politique à l'horizon de cela... Par exemple, dans Je suis une aventure, il ne s'agit pas d'un corps rabelaisien, puisque le corps d'un sportif comme Federer c'est justement l'inverse d'un corps rabelaisien. Mais pourtant, c'était pour moi le même travail : ce que je voulais faire avec le corps de Federer, c'était montrer qu'un corps qui parvient à être pleinement présent à tout ce qui s'offre à lui à un moment donné, donc qui parvient à être créateur, créatif, c'est justement un corps qui permet de réinventer complètement la notion d'identité. On n'est pas tout d'un coup prisonnier de ce qu'on est - Roger Federer plus grand joueur de l'histoire, etc.: non, au moment où il invente, il est dans une espèce d'état de grâce. L'état de grâce, c'est arriver à faire vivre absolument toutes les possibilités du moment présent, arriver à bricoler avec, ne pas devoir appliquer une recette. Donc je peux aller du corps disons protorabelaisien dans Appoggio au corps de Federer qui semble être un corps en contrôle, mais à chaque fois c'est la même chose, c'est essayer de sortir de l'identité, telle qu'on la vit nous ici, c'est-à-dire l'identité qui est une forme de prison. Ce n'est pas le corps pour moi qui est une prison, c'est beaucoup plus une façon de se penser, de représenter, de dire : «Voilà, bonjour, je suis écrivain, etc. ». Pour moi, c'est cent fois plus inquiétant que les limites du corps.

\section{NOTES}

1. Arno Bertina, La Déconfite gigantale du sérieux, Paris, Léo Scheer, coll. "Lignes Manifestes ", 2004, p. 68.

2. Ibid., p. 52-53.

3. Arno Bertina, Appoggio, Arles, Actes Sud, 2003, p. 64-66. 


\section{RÉSUMÉS}

Entretien avec Arno Bertina, réalisé le 4 avril 2016 à la Maison des Sciences de l'Homme de Clermont-Ferrand, dans le cadre du festival LAC 2016.

Interview with Arno Bertina, April 4th 2016 at the Maison des Sciences de l'Homme of ClermontFerrand, during the LAC festival 2016.

\section{INDEX}

Keywords : Appoggio, La Déconfite gigantale du sérieux, Body

Mots-clés : Appoggio, La Déconfite gigantale du sérieux, Corps

\section{AUTEURS}

\section{ARNO BERTINA}

Romancier du fait social, historique, politique, Arno Bertina goûte également les délices et excès rabelaisiens, comme dans La Déconfite gigantale du sérieux. Cette autobiographie imaginaire peuplée de personnages à la langue débraillée fait l'éloge d'une littérature nourrie et copieusement vivante. Bibliographie : La déconfite gigantale du sérieux, Lignes-Léo Scheer, 2004. Numéro d'écrou 362573, avec la photographe Anissa Michalon, Le Bec en l'air, 2013. Des châteaux qui brûlent, Verticales, 2017.

\section{JOHAN FAERBER}

Dans son anthologie La cuisine des écrivains, Johan Faerber, essayiste et critique littéraire, propose un savoureux voyage à travers les siècles, à la rencontre des écrivains qui ont célébré la nourriture et la boisson.

\section{NATHALIE VINCENT-MUNNIA}

Maîtresse de conférences en littérature française du XIX siècle à l'université Clermont Auvergne, elle a travaillé sur la poésie en prose au XIX ${ }^{\mathrm{e}}$ siècle, et traite plus largement les problématiques génériques et littéraires liées aux marges, frontières, altérités, à partir du XIX siècle : questions socio-esthétiques des marginalités, poésie ouvrière et populaire, poésie et discours politiques, artistiques, critiques. Elle effectue également des travaux dans le domaine de l'action culturelle, des formations artistiques, des métiers du livre, des arts, et de la culture. Elle a notamment publié Les premiers poèmes en prose : généalogie d'un genre dans la première moitié du XIXe siècle français (Champion, 1996). 